\title{
Commentary: The Dynamics of Aerotaxis in a Simple Eukaryotic Model
}

\author{
Jean-Paul Rieu ${ }^{1 *}$, Olivier Cochet-Escartin ${ }^{1}$, Christophe Anjard ${ }^{1}$, Mete Demircigil ${ }^{2}$ and \\ Vincent Calvez ${ }^{2}$
}

${ }^{1}$ Institut Lumière Matière, UMR5306, Université Lyon 1-CNRS, Université de Lyon, Villeurbanne, France, ${ }^{2}$ Institut Camille Jordan, UMR5208, Université Lyon 1-CNRS, Université de Lyon, Villeurbanne, France

Keywords: Self-generated gradients, Aerotaxis, Collective migration, Oxygen sensing, Dictyostelium discoideum

\section{A Commentary on}

The Dynamics of Aerotaxis in a Simple Eukaryotic Model

by Biondo, M., Panuzzo, C., Ali, S. M., Bozzaro, S., Osella, M., Bracco, E., and Pergolizzi, B. (2021). Front. Cell Dev. Biol. 9:720623. doi: 10.3389/fcell.2021.720623

We read with interest the article by Biondo et al. (2021) in Frontiers in Cell and Developmental Biology,

OPEN ACCESS

Edited by:

Verena Ruprecht,

Centre for Genomic Regulation (CRG),

Spain

Reviewed by:

Jean Clairambault,

Institut National de Recherche en

Informatique et en Automatique

(INRIA), France

*Correspondence:

Jean-Paul Rieu

jean-paul.rieu@univ-lyon1.fr

Specialty section:

This article was submitted to

Molecular and Cellular Pathology,

a section of the journal

Frontiers in Cell and Developmental

Biology

Received: 28 December 2021 Accepted: 20 January 2022 Published: 02 March 2022

Citation:

Rieu J-P, Cochet-Escartin O, Anjard C,

Demircigil M and Calvez V (2022)

Commentary: The Dynamics of

Aerotaxis in a Simple

Eukaryotic Model.

Front. Cell Dev. Biol. 10:844812.

doi: 10.3389/fcell.2022.844812
"The Dynamics of Aerotaxis in a Simple Eukaryotic Model." Reproducing the confinement assay we published in eLife earlier this year (Cochet-Escartin et al., 2021) with the same cell line, they found the same emergent behavior, i.e., the propagation of a ring of cells, which they named corona, from a dense, confined colony through the self-generation of oxygen gradients by cell consumption. The authors claimed that cell division plays no role in the phenomenon, whereas in our study, we insisted on its important role.

This message is wrong. In this commentary, we first clarify that ring formation is independent on cell division but that ring propagation over long times depends on it. Second, we discuss the possible experimental biases that may have led the authors to this conclusion.

Cell division is not necessary for ring formation but is necessary for its sustained propagation. Biondo et al. observed exactly the same collective phenotype as us for the confined colony in a starving buffer that prevents cell division. A ring forms internally, but as soon as it reaches the colony edge (at $\sim 6 \mathrm{~h}$ ), it stops and cells aggregate (compare Movie 3 of Biondio et al. with our movie M6 and Figure 5; Supplementary Figure 2, Cochet-Escartin et al.). In contrast, in a nutrient medium, the ring propagates far away from the initial colony for days (see Figure 1A below). Biondo et al. neither commented on this fundamental difference between the two conditions nor on our model that demonstrates that cell division is necessary to maintain ring propagation even if it contributes little to the expansion speed (Figures $5 \mathrm{~A}, \mathrm{~B}$ and Eq. 6, Cochet-Escartin et al.). Independently of any model, a simple mass balance equation for the total cell number $\mathrm{N}$ with NB cells in the bulk region (core) and NR cells in the ring region invalidates Biondo's assertion that division plays no role:

$$
N(t)=N_{B}(t)+N_{R}(t)=\rho_{B} \pi(R(t)-L)^{2}+\rho_{R} 2 \pi R(t) L
$$

Using the experimental observations (Figures 1D,E, Supplementary Figure S3B in Cochet-Escartin et al., Figures 1B-D below) that the ring width $L$ and density $\rho_{R}$ and bulk density $\rho_{B}$ are constant, and that the ring radius $\mathrm{R}$ is expanding at constant speed $R(t)=R_{0}+\sigma t$, we predict that $N_{B}(t)$ increases faster with time (i.e., as $R^{2} \sim t^{2}$ ) than $N_{R}(t)$ (i.e., as $L R \sim t$ ). Experimentally, up to $30 \mathrm{~h}, N_{B}(t)$ increases faster than linearly with time while $N_{R}$ increases linearly (Figure 1C). Initially $N_{B} / N_{R}=1$, but after $24 \mathrm{~h}$, $N_{B} / N_{R}=1.8$, and after $47 \mathrm{~h}, N_{B} / N_{R}=2.8$ (Figure 1C). Hence, $N_{B}$ largely contributes to the overall cell number increase $N(t)$. By comparison, Biondo et al. assume a constant $N_{R}(t)$, and they do not consider bulk cells at all. 

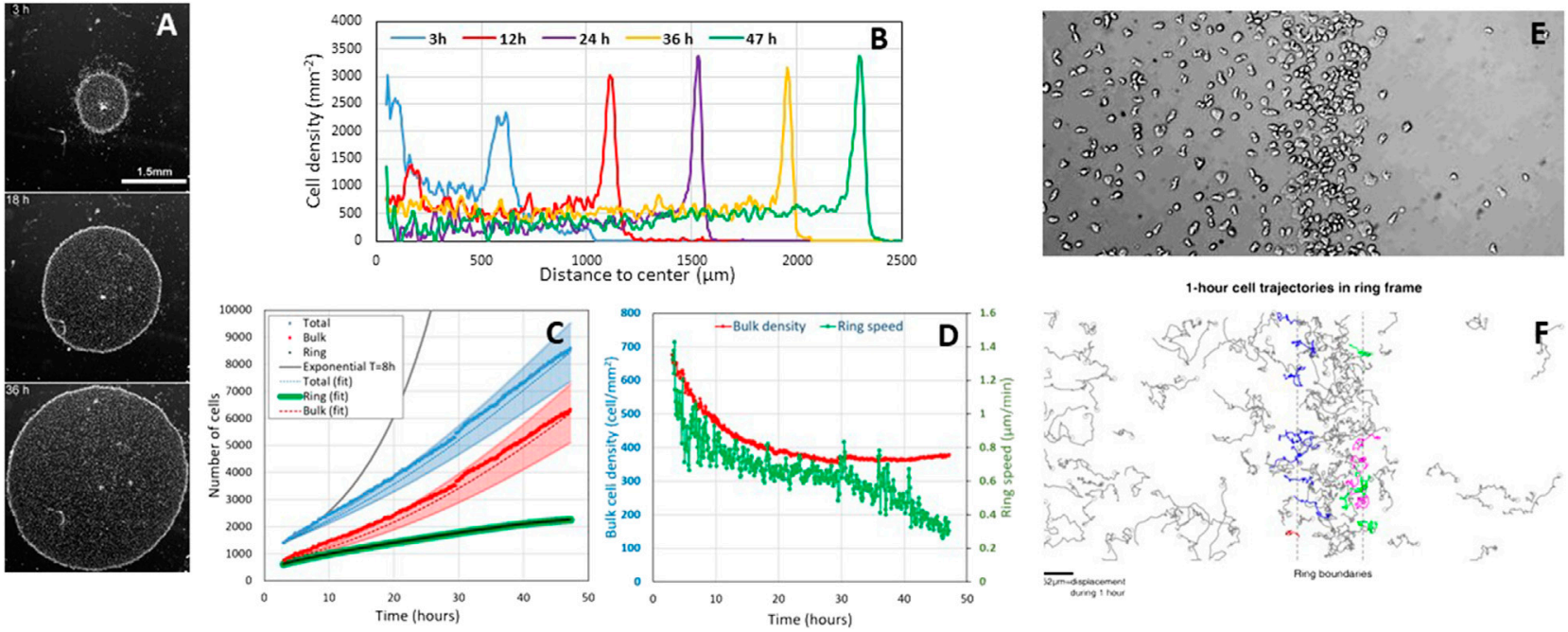

FIGURE 1 | Cell proliferation during the aerotactic expansion of a small confined colony of vegetative Dictyostelium cells with an initial number of cell $N(0 \mathrm{~h})=1,000$ and an initial radius $R(0 \mathrm{~h})=600 \mu \mathrm{m}$. (A) Snapshots at 3, 18, and $36 \mathrm{~h}$ showing the propagation of an external dense ring of cells mowing outwardly. Scale bar, $1.5 \mathrm{~mm}$. (B) Corresponding stationary radial density profiles. (C) Measurements of the number of ring and bulk cells as well as the sum of the two subpopulations (total). The ring cells were estimated by measuring their density per unit length and multiplying by the perimeter. The bulk and total cell numbers have been fitted by Eqs $\mathbf{1}, \mathbf{2}$ (dotted lines) with $\varphi_{R \rightarrow B}=12 \mathrm{cell} / \mathrm{s} / \mathrm{mm} / \mathrm{h}$ and error bars correspond to a $20 \%$ error on $\varphi_{R \rightarrow B}$. (D) Ring speed and bulk cell density measurements over time. (E,F) Close view of the ring region to estimate the cell exchanges between the ring and the bulk. (F) Cell trajectories lasting $1 \mathrm{~h}$ in the ring frame. Ring borders are depicted by the dashed line. Blue, red, green, and purple trajectories correspond to trajectories that escape the ring toward the bulk, reach the ring from the bulk, reach the ring from the front, and escape the ring from the front, respectively. Overall, the net flux of cell from ring to front is zero; the net flux of cell from ring to bulk is approximately $\varphi_{R \rightarrow B}=12 \mathrm{cells} / \mathrm{mm} / \mathrm{h}$

Cell divisions hold in the ring. Our confined colony grows slower than exponentially (see solid black line with a typical $8 \mathrm{~h}$ doubling time (d'Alessandro et al., 2016) in Figure 1C), but it grows (i.e., $N(47 \mathrm{~h}) / N(0 \mathrm{~h})=8.5)$. In Cochet-Escartin et al., we propose a go-or-grow model where aerotaxis holds at low $\mathrm{O}_{2}$ and cell division at high $\mathrm{O}_{2}$. The threshold is around $1 \% \mathrm{O}_{2}$ as estimated by direct aerotaxis investigations using microfluidic devices (Cochet-Escartin et al.) and from literature values for cell division in hypoxic conditions (Schiavo and Bisson, 1989; West et al., 2007). Such value corresponds to the $\mathrm{O}_{2}$ level measured in the ring (Cochet-Escartin et al.). Hence, divisions occur mostly in the ring, but ring cells are constantly transferred to the bulk to maintain a constant $\rho_{B}$ while $R$ is increasing. This transfer occurs in our models (see Figure 7 of Cochet-Escartin et al.), but perhaps it was not sufficiently supported by data. In Figures 1E,F, we present manually tracked trajectories in the ring frame. A few ones displayed with a green or purple color enter or escape the outward ring position, canceling any ring-to-front flux. Far more trajectories are directed backward (i.e., ring to bulk, in blue). Interestingly, the measured flux of such a cell transfer, $\varphi_{R \rightarrow B}=12$ cells $/ \mathrm{mm} / \mathrm{h}$, explains fairly well the bulk cell number increase using the following equation:

$$
d N_{B}=2 \pi R \phi_{R \rightarrow B} d t
$$

The fit is displayed in Figure 1C.

Biondo et al. may have caught a transient regime only. Biondo et al. measured 3\% and 5\% $\mathrm{O}_{2}$ in the bulk and ring regions, respectively (Supplementary Figure $\mathrm{S} 1$ ). Above $2 \% \mathrm{O}_{2}$, aerotaxis should not hold (Cochet-Escartin et al.); the division rate is fairly the same as in normoxic conditions (Schiavo and Bisson, 1989; West et al., 2007). A possible reason for this discrepancy is that $\mathrm{O}_{2}$ is overestimated. Their measurements were performed with a commercial sensing film that is not compatible with transmission microscopy, contrary to the technology we developed in Cochet-Escartin et al. They may have a different confinement on plastic (their usual experimental condition) than on the sensing film. A loose (resp. tight) confinement may generate a higher (resp. lower) $\mathrm{O}_{2}$ value under the colony. They also made colonies with a huge amount of cells $(50,000$ instead of 1,000 and 2,000 in our case). As the self-generated $\mathrm{O}_{2}$ field depends on the consumption of every cell, we expect a huge degree of hypoxia. Finally, they never reached a stationary expansion regime due to the large initial excess of inner cells. That excess density slowly decreases with time as visible on their kymograph. We have actually simulated a moderate bulk cell excess in our work (Figure 4 of Cochet-Escartin et al.) which is also transiently visible at $3 \mathrm{~h}$ in Figure 1B. Such an inner cell mass transfer has to be taken into account to establish a correct mass balance equation, and the only $L(t) R(t)$ quantity tested by Biondo et al. is clearly not sufficient to draw a conclusion on cell divisions.

\section{AUTHOR CONTRIBUTIONS}

All authors listed have made a substantial, direct and intellectual contribution to the work, and approved it for publication. 


\section{FUNDING}

This study was supported by the CNRS - Mission pour les Initiatives Transverses et Interdisciplinaires' "Défis Modélisation du vivant - 2019", by the European Research

\section{REFERENCES}

Biondo, M., Panuzzo, C., Ali, S. M., Bozzaro, S., Osella, M., Bracco, E., et al. (2021). The Dynamics of Aerotaxis in a Simple Eukaryotic Model. Front. Cel Dev. Biol. 9, 720623. doi:10.3389/fcell.2021.720623

Cochet-Escartin, O., Demircigil, M., Hirose, S., Allais, B., Gonzalo, P., Mikaelian, I., et al. (20212021). Hypoxia Triggers Collective Aerotactic Migration in Dictyostelium discoideum. eLife 10, e64731. doi:10.7554/eLife.64731

d'Alessandro, J., Mas, L., Aubry, L., Rieu, J. P., Rivière, C., and Anjard, C. (2018). Collective Regulation of Cell Motility Using an Accurate Density-Sensing System. J. R. Soc. Interf. 15, 45. doi:10.1098/rsif.2018.0006

Schiavo, G., and Bisson, R. (1989). Oxygen Influences the Subunit Structure of Cytochrome C Oxidase in the Slime Mold Dictyostelium discoideum. J. Biol. Chem. 264, 7129-7134. doi:10.1016/s0021-9258(18)83211-2

West, C. M., van der Wel, H., and Wang, Z. A. (2007). Prolyl 4-hydroxylase-1 Mediates O2 Signaling during Development ofDictyostelium. Development 134, 3349-3358. doi:10.1242/dev.000893
Council (ERC) under the European Union's Horizon 2020 research and innovation program (grant agreement No 865711 to VC) and by the International Human Frontier Science Program Organization, Grant Number RGP0051/2021 (to J-PR).

Conflict of Interest: The authors declare that the research was conducted in the absence of any commercial or financial relationships that could be construed as a potential conflict of interest.

Publisher's Note: All claims expressed in this article are solely those of the authors and do not necessarily represent those of their affiliated organizations, or those of the publisher, the editors and the reviewers. Any product that may be evaluated in this article, or claim that may be made by its manufacturer, is not guaranteed or endorsed by the publisher.

Copyright (C) 2022 Rieu, Cochet-Escartin, Anjard, Demircigil and Calvez. This is an open-access article distributed under the terms of the Creative Commons Attribution License (CC BY). The use, distribution or reproduction in other forums is permitted, provided the original author(s) and the copyright owner(s) are credited and that the original publication in this journal is cited, in accordance with accepted academic practice. No use, distribution or reproduction is permitted which does not comply with these terms. 